\title{
Geopolítica en América del Sur: Desde la militarización de la disciplina a la necesidad del debate académico
}

\author{
Geopolitics in South America: \\ From the militarisation of discipline to the need for academic debate \\ LESTER CABRERA TOLEDO ${ }^{1}$ \\ FLACSO, Ecuador
}

RECEPCIÓN: 26/09/2017 • ACEPTACIÓN: 23/10/2017

RESUMEN: El presente artículo tiene por objetivo analizar algunos de los factores que han llevado a la utilización de la geopolítica en América del Sur como un concepto amplio y vago que busca explicar algunos procesos internacionales. Para lograr lo anterior, al mismo tiempo que se problematiza a la geopolítica desde su concepto, se realizará una descripción histórica sobre su evolución en la región mencionada, tomando en cuenta tanto el contexto regional, la percepción geopolítica de algunos actores, como también el papel que jugaron los militares en el desarrollo de la disciplina, especialmente durante la Guerra Fría. Así también, se tomarán en cuenta algunos de los elementos que establecen la necesidad de aumentar el debate dentro de la disciplina, desde la óptica de la universidad, producto de la existencia de aspectos que desde la visión "tradicional" de la disciplina, no se pueden entender, tales como la identidad y la visión de espacio. Se concluye que resulta necesario para el desarrollo de la disciplina en la región, un debate entre la academia y los estamentos armados en la disciplina de la

1. Licenciado en Ciencias Políticas y Administrativas, y Administrador Público con mención en Ciencia Política por la Universidad de Concepción. Diplomado en Estudios Políticos y Estratégicos, y Magíster en Seguridad y Defensa con mención en Política de Defensa, por la Academia Nacional de Estudios Políticos y Estratégicos. email: lecabrerafl@flacso.edu.ec 
geopolítica, para tener una mejor comprensión de lo que se entiende, y lo que no se entiende, por geopolítica.

PALABRAS CLAVE: Geopolítica - Sudamérica - disciplina - Fuerzas Armadas.

ABSTRACT: The object of the present article is to analyse some of the factors that have led to the use of geopolitics in South America as a broad, vague concept intended to explain certain international processes. To do this we seek to problematize geopolitics from its concept; and in parallel we present a historical description of the evolution of the discipline in the Region, taking into account the regional context, the geopolitical perception of some of those involved, and the role played by the military in its development - especially during the Cold War.We will also consider some of the elements that determine the need to increase debate within the discipline from the universityperspective, since there are certain aspects - such as identity and the perspective of space- that cannot be understoodfrom the "traditional" viewpoint. We conclude that the development of the discipline in the region requires debate about geopoliticsbetween the academicsphere and the Armed Forces, to obtain a better understanding of what is, and what is not, understood by the term.

KEYWORDS: Geopolitics - South America - discipline - ArmedForces

\section{Introducción}

¿Por qué la geopolítica es un concepto tan amplio y vago que ayudaría a entender una buena parte de los conflictos internacionales? ¿Cuáles son los elementos que permiten entender aquella amplitud conceptual, especialmente en América del Sur? Este artículo tiene por objetivo responder a estas interrogantes, tomando en cuenta algunos debates académicos que se han venido dando en la región señalada, sobre la base de la evolución de la disciplina. Pero sin perjuicio de un posterior desarrollo de las eventuales respuestas que se darán, desde un punto de vista metodológico, resulta adecuado dar luces sobre las interrogantes planteadas, con un objetivo meramente introductorio. La vaguedad y amplitud de la geopolítica, como un concepto que se utiliza casi de manera indiscriminada, para dar una explicación sobre los fenómenos conflictivos, tiene una raíz tanto epistemológica como teórica. En este plano, la conceptualización de la geopolítica puede tener una interpretación tanto desde la óptica de la geografía, como también de la ciencia política, los estudios 
internacionales, y sus diferentes ramificaciones disciplinarias. Por ende, es posible hablar de geopolítica, tomando en cuenta múltiples puntos de vista. Y si a lo anterior se añade que la concepción teórica de la geopolítica es difusa, generalmente asociándose de manera errónea con una perspectiva realista, deja una visión sesgada sobre sus postulados y formas de explicar fenómenos.

Lo mencionado, es decir, esa carencia de claridad epistémica y teórica que deriva en una amplitud y vaguedad conceptual aplicado al caso de América del Sur, tiene también una eventual respuesta en lo que se podría denominar una "militarización” de la geopolítica. Desde incluso antes de la Guerra Fría, los conceptos y lineamientos geopolíticos generados en Europa, y especialmente aquellos que tienen un origen alemán, decantaron en América del Sur por medio de los Oficiales de enlace de los países latinos en Europa. Es decir, fueron las Fuerzas Armadas las receptoras directas del conocimiento geopolítico, y especialmente en las Escuelas Superiores o Academias de Guerra de los ejércitos. Y derivado de la estigmatización de la geopolítica por parte de los aliados, dicho conocimiento estuvo circunscrito de manera casi exclusiva a dichos cuerpos y estamentos, adquiriendo una connotación nacional a través de la aplicación de los lineamientos geopolíticos a las circunstancias propias de cada país. Sin embargo, la concepción del pensamiento geopolítico no tuvo variación alguna, especialmente desde la óptica epistémica y teórica, ni tampoco una respuesta de parte de la academia. Por lo tanto, los parámetros iniciales se siguieron replicando a lo largo de los años, no permitiendo un desarrollo o respuesta externa a lo militar.

El objeto de estudio se enfoca en describir cómo ha sido el proceso de desarrollo y evolución de la geopolítica en Sudamérica, tomando como base una utilización superficial y amplia del concepto, así como también el rol de los militares y la academia. El papel que ha jugado el sector militar en el ámbito de la geopolítica, si bien ha sido considerado en cuanto a sus postulados, su visualización y contribución al debate disciplinario, no posee el mismo nivel de observación. Mientras que por otro lado, se considerará el aporte de la academia en el debate de la geopolítica en la región, tanto para contrastar como también para lograr una explicación al fenómeno descrito en su momento.

De lo señalado, es posible establecer que la principal contribución del manuscrito, más allá de ayudar a clarificar el concepto de geopolítica, es comprender algunos de los factores y causas que, de alguna u otra forma, determinan la utilización vaga y amplia del concepto de geopolítica, en lo relativo al contexto sudamericano. La selección de dicho espacio geográfico se da, porque es en dicha área del planeta, en que la visión y utilización de la geopolítica se mantuvo, incluso en períodos de la 
historia en los que la geopolítica era considerada como una rama del conocimiento negativa y cargada de un estigma ideológico, como consecuencia de los sucesos dados en la Segunda Guerra Mundial, específicamente desde el lado nazi.

Se concluye que si bien la región señalada ha sido un espacio en donde la geopolítica se ha desarrollado, especialmente en aquellos años en los cuales se le consideraba como un área del conocimiento relacionada al nazismo, aquel desarrollo en la actualidad no puede estar circunscrito exclusiva o de manera mayoritaria a los círculos militares, sino que la academia debe cumplir un rol integrador que permita llevar el debate más allá de las concepciones clásicas derivadas del pensamiento del siglo XIX. En este sentido, lo anterior puede incluso concebirse y construirse como un canal de comunicación entre civiles y militares, tomando como base la generación de un conocimiento que ayude al desarrollo de los diferentes países, e incluso a la región en términos generales.

\section{La geopolítica como problema conceptual}

Es sumamente recurrente, tanto en el ámbito de los medios de comunicación masivos, como también en determinadas revistas y libros vinculados a la academia, visualizar la forma en que la geopolítica es utilizada de una manera vaga, como una forma de explicar diferentes fenómenos sociales, tanto nacionales como internacionales, que por lo general se relacionan con un acontecimiento conflictivo. Es así como las diferentes crisis económicas o del petróleo, tienen un eventual origen geopolítico. Lo mismo sucede cuando un país está en proceso de adquirir armas nucleares, o bien cuando hay intentos de secesión de un área territorial que desea autonomía. Siendo así, resulta necesario no solamente otorgar un punto de partida para poder entender qué es lo geopolítico, o incluso mejor, para saber qué no es lo geopolítico. Pero incluso para lograr dicho objetivo, las visiones y concepciones de la disciplina instalan un panorama altamente complejo.

Sobre lo mencionado, es posible encontrar múltiples ejemplos, incluso dentro del plano académico. Phil Kelly en su reciente obra Classical Geopolitics: a new analytical model, señala explícitamente que el debate en torno a encontrar un modelo de análisis "geopolítico", es tremendamente complejo, producto de que no se tiene una concepción clara de lo que es la geopolítica. Solamente se tiene una visión de lo que "puede ser" 2 . Otro ejemplo de lo anterior es lo que plantea Saul Bernard Cohen, quien señala que incluso no es posible catalogar a la geopolítica

\footnotetext{
2. KelLy (2016), p. 9.
} 
como parte de un conocimiento exclusivo, ya sea de la geografía humana o la ciencia política ${ }^{3}$. Por ende, la geopolítica como concepto representa un problema que deber ser considerado y explicado desde diferentes puntos de vista.

En primer lugar, es necesario señalar que el hecho de establecer una definición exclusiva de la geopolítica, implica la reducción de una serie de elementos que, de alguna u otra forma, pueden intervenir en el entendimiento del proceso o fenómeno que se denomina geopolítico. Es así como hay autores que establecen un predominio de lo internacional por sobre lo nacional ${ }^{4}$, otros que enfatizan la cualidad geográfica del fenómeno por sobre el entendimiento político ${ }^{5}$, mientras que otros otorgan un valor especial a los procesos económicos, sociales y culturales como parte de un entendimiento geopolítico a un determinado evento ${ }^{6}$. Pero sin perjuicio de los múltiples puntos de vista que se pueden recabar del mismo concepto, lo cierto es que se puede visualizar un común denominador en la totalidad de las definiciones, y es que la geopolítica, pese a las variantes epistémicas, incluye una concepción territorial. Es así que tanto para las corrientes positivistas como para las interpretativistas, si bien es cierto que hay aspectos en los cuales hay mayor importancia que otros, la concepción de territorio y/o espacio es algo que está presente.

En segundo lugar, y como ya se mencionó, no resulta posible hablar de una exclusiva geopolítica, más allá de que se ponga énfasis en determinados ámbitos disciplinarios. La concepción epistemológica de la geopolítica no es única. La evolución del pensamiento en las Ciencias Sociales, trajo como consecuencia un impacto en la geopolítica, estableciéndose una división entre el pensamiento clásico, eminentemente positivista, y la concepción crítica de la geopolítica, la cual tiene una raíz epistémica diferente. Así, algunas de las principales diferencias radican tanto en los niveles de análisis, como en la misma finalidad de aquellas concepciones ${ }^{7}$. Por ejemplo, desde el ámbito de los niveles de análisis, la geopolítica clásica se enfoca en tomar en cuenta cómo la estructura influye en los diferentes agentes, siguiendo con ello la lógica del pensamiento positivista ${ }^{8}$; mientras que la visión crítica de la geopolítica pone énfasis en los diferentes procesos de toma de decisión, lo que podría
3. COHEN (2015), p. 15.
4. Flint (2006), p. 13.
5. KAPLAN (2012), p. 11.
6. O’TUATHAil y AGNEW (1992), p. 192-193.
7. Cabrera (2017), p. 120-121.
8. CoHen (2015), p. 16. 
denominarse "agencia”, en un lenguaje constructivista9. Por lo tanto, un mismo acontecimiento geopolítico puede tener una interpretación diferente, dependiendo del enfoque epistémico con el cual se analice y explique.

$\mathrm{Y}$ en tercer lugar, existe una dificultad al momento de establecer el objeto de estudio de la geopolítica. Para las concepciones clásicas de la disciplina, el actor principal de la geopolítica es el Estado y la interacción que este posee con otros actores similares. Es decir, las eventuales explicaciones de fenómenos geopolíticos, si bien tienen un origen territorial, decantan en una ubicación geográfica del Estado con respecto al contexto internacional. Incluso, lo anterior queda supeditado a las acciones que realicen otros países, tomando en consideración la naturaleza sistémica del contexto internacional, como bien lo señalan Peter Taylor y Colin Flint ${ }^{10}$. Mientras que para otros autores, el objeto de estudio de la geopolítica no puede circunscribirse solamente al Estado, debido a que existen una serie de fenómenos territoriales que no pueden entenderse desde la óptica exclusiva de la institución estatal. Aspectos tales como los símbolos, las identidades, los discursos e incluso determinados actores locales y regionales, se hacen invisibles a través de la figura del Estado. Con ello, la geopolítica debe tener un objeto de estudio más amplio que el Estado ${ }^{11}$.

Siendo así, la conformación y estructuración de un proceso geopolítico, decanta de manera directa en la forma en cómo se analice, de acuerdo a los actores y factores involucrados. Pero lo cierto es que existen una serie de aspectos, especialmente el territorial-espacial, que no se puede dejar de lado, si se realiza una explicación en torno a un fenómeno, caracterizado como geopolítico. El territorio-espacio es el elemento principal que determina si se está en presencia de un acontecimiento geopolítico o no. Sin embargo, aquella visión sigue siendo sumamente amplia, por lo que necesariamente el territorio-espacio se debe complementar con una perspectiva política. De este modo, la geopolítica implica un proceso en el cual se hace una interpretación de características políticas sobre los medios que se encuentran ubicados en un territorio-espacio determinado y, por lo general, aquella interpretación posee consecuencias internacionales. Así, si bien es cierto que pueden considerarse como nacionales ciertos procesos geopolíticos, estos no podrían ser catalogados como tales, si no poseen una mirada o consecuencia internacional ${ }^{12}$.

9. FonT y Rufí (2001), p. 29-30.

10. TAYLOR y Flint (2002), p. 102.

11. MÜller (2008), p. 323-325.

12. FuINT (2006), p. 17. 
La problemática señalada, es decir, la interpretación de un proceso geopolítico como tal, con respecto a la conceptualización de la geopolítica, es que es un debate que ha sido guiado por lineamientos de la academia, pero ubicados geográficamente en el hemisferio norte, en donde se posee una visión más amplia de la disciplina. Para el caso de América Latina, ocurren dos fenómenos que ayudarían a explicar el porqué aquellos debates no se dan; o si se dan, no con la suficiente profundidad. En primer lugar, el conocimiento geopolítico en las diferentes universidades no es relativamente conocido o debatido, debido al mismo desconocimiento que se tiene de la materia. Y en segundo lugar, el conocimiento geopolítico ha sido administrado especialmente por las Fuerzas Armadas de la región, desarrollándose de manera más amplia en las Escuelas Superiores o Academias de Guerra, donde se forman los Oficiales de Estado Mayor. Por lo tanto, los debates en el plano conceptual y todo lo que aquello involucra, no es propio ni de la profesión militar ni ha sido objeto de una discusión académica en forma regular a nivel de las universidades.

Pero sin perjuicio de lo anterior, la región tiene una tradición, no muy reconocida, en la mantención y desarrollo del concepto, en comparación a otras áreas geográficas del planeta ${ }^{13}$. Se establecieron "escuelas geopolíticas” de pensamiento, y durante algún tiempo, escritores de obras geopolíticas plasmaron su pensamiento en la política interna y exterior de los países de la región. Es así que el contexto mencionado, en conjunto con otros elementos, ayudaría a explicar la condición actual de la disciplina en la región señalada.

\section{La geopolítica en América Latina durante la Guerra Fría}

Como ya se mencionó, la geopolítica en la región ha sido uno de los elementos que más fuertemente se ha estudiado, en comparación con otras regiones. Incluso, autores como Phil Kelly ${ }^{14}$ y Klaus Dodds ${ }^{15}$ señalan que América Latina fue un baluarte para la mantención y desarrollo del conocimiento geopolítico, especialmente en momento en donde el mundo académico de los países aliados, post Segunda Guerra Mundial, consideraban a la geopolítica como una "ciencia nazi". En este plano, ambos autores destacan que la geopolítica en América Latina tuvo un gran impacto en los diferentes proyectos desarrollistas y de política exterior, especialmente en el nivel

13. Kelly (2016), p. 6.

14. Kelly (2016), p. 14-15.

15. Dodds (1993), p. 361. 
vecinal y regional. Sin embargo, fue por medio de los segmentos militares de los países latinos, que el conocimiento geopolítico se desarrolló. Este aspecto, a juicio de Klaus Dodds, fue uno de los elementos que estableció una lógica imperialista y bélica en el pensamiento geopolítico latinoamericano, visualizándose tanto en publicaciones de autores militares, como también en la forma en que las dictaduras de la región, alrededor de la década de los 70, plasmaron la visión geopolítica en sus proyectos de país y, especialmente, en sus políticas exteriores ${ }^{16}$.

Siguiendo aquella condición de "excepcionalidad" del conocimiento geopolítico en América del Sur, en relación a otras regiones en plena época de la Guerra Fría, dicha condición también posee una connotación altamente ideológica en el uso del concepto como tal; no obstante, la conformación de determinados parámetros para comprender la eventual expansión de los Estados, o en su defecto la mantención de espacios hegemónicos y de dominación por aquellos años, fue una realidad. Al respecto, los proyectos de países con intereses a nivel mundial, siguiendo con ello la visión de los "códigos geopolíticos" 17 , si bien no empleaban el concepto "geopolítica", hacían alusiones similares a las que propugna la disciplina. Uno de los ejemplos más recordados es lo que planteó en su momento George Kennan con su "teoría del dominó" 18 . Y fue recién desde la década de los 70 , en que el concepto fue nuevamente aludido como forma de explicación de la política mundial, a través de Henry Kissinger y su obra La Diplomacia ${ }^{19}$. Pero también al respecto, es necesario resaltar los esfuerzos de franceses como Yves Lacoste, o la revista Herodoto en dicho país, para resaltar la importancia y utilización del concepto, más allá de la instrumentalización negativa que tuvo en el pasado ${ }^{20}$.

Pero sin perjuicio de lo acontecido en el contexto mundial, la concepción geopolítica de la época no puede desligarse del contexto en el cual Sudamérica se encontraba inmersa. Siendo así, los proyectos desarrollistas impulsados por la CEPAL, las consecuencias del conflicto bipolar que tenía alcance planetario, manifestándose principalmente en la región a través de diversas revoluciones, como también la visión geopolítica de Estados Unidos, basada en la mantención de una hegemonía hacia América Latina en temas políticos, económicos y militares, fueron aspectos que

16. DodDs (1993), p. 363 .

17. FLINT (2006), p. 58-59.

18. CoHen (2015), p. 29.

19. O’tuathail (1996), p. 45.

20. Lacoste (2011), p. 17-21. 
tuvieron consecuencias en la composición del pensamiento geopolítico de la región. En este sentido, la geopolítica tuvo una utilización altamente vinculada con los diferentes proyectos de desarrollo que emergían en la región, lo que a su vez la hacia presa de una concepción altamente sesgada con los intereses no necesariamente de un país, sino que de una cúpula que poseía un elevado grado de control político en la sociedad ${ }^{21}$. Es así que, dentro del plano teórico, a la geopolítica en Sudamérica se le asocia en términos de una vertiente del realismo político, tomando en consideración el hecho de que tanto para la concepción realista como para la geopolítica, el actor central de análisis radica en el Estado y su eventual supervivencia ${ }^{22}$.

Como consecuencia del contexto mencionado, tanto en el plano teórico como también de la coyuntura política de la segunda mitad del siglo XX, es que la geopolítica en América Latina, y específicamente en América del Sur, ha sido uno de los conceptos más estudiados desde la óptica nacional. Es así que autores tales como John Child y Klaus Dodds señalan la existencia de tres grandes expresiones del pensamiento geopolítico en la región, las cuales denominan "escuelas de pensamiento geopolítico", circunscribiendo a Brasil, Argentina y Chile. No obstante, aquello no descarta la existencia de un pensamiento geopolítico en otros países de la zona, pero son los Estados señalados los que marcaron la pauta en la composición de lineamientos teóricos aplicables a sus realidades nacionales ${ }^{23}$.

Al hablar de "escuelas de pensamiento", si bien autores como John Child, Phil Kelly o Jonathan Barton han destacado la existencia de un pensamiento geopolítico en América Latina, no señalan de manera explícita lo que conlleva a la creación de una escuela de pensamiento. Así, para Child, el establecimiento de una "escuela de pensamiento" en el ámbito de la geopolítica, estaría determinada por el grado de desarrollo de "teorías geopolíticas", aplicables a una realidad nacional en cuestión. Esto, sumado a su grado de ejecución dentro de un período de la historia, serían patrones que el mencionado autor tomaría en cuenta para señalar la existencia de una "escuela geopolítica" 24 . Y tanto Phil Kelly como Jonathan Barton, mencionan la importancia del pensamiento geopolítico, especialmente en el Cono Sur de Sudamérica, para explicar determinados lineamientos asociados a la planificación y posterior ejecución de las políticas exteriores ${ }^{25}$.

21. BARTON (1997), p. 49-50.

22. COHEN (2015), p. 81-82.

23. CHILd (1979), p. 89; DodDs (1993), pp. 363-365.

24. Child (1979), p. 89.

25. Véase al respecto Kelly (2016) p. 6; y Barton (1997), p. 14. 
Uno de los elementos que también se aprecian dentro del pensamiento geopolítico en Sudamérica, cuya base teórica y conceptual fue construida mayormente durante la época de la Guerra Fría, es la explicación de los diferentes conflictos territoriales que se han suscitado desde la constitución de los Estados-Nación. Es así como, por ejemplo, que las desavenencias territoriales, así como también las eventuales pérdidas y ganancias de territorio por parte de los Estados, se encuentran dentro del esquema de pensamiento de los principales autores militares que tratan la disciplina de la geopolítica. Pero en este plano, existen diferencias en torno a la postura de los diferentes países de la región. Por ejemplo, en el caso de la escuela de pensamiento geopolítico de Brasil, no solamente se aspiraba a tener un control efectivo de su vasto territorio y de sus miles de kilómetros de frontera ${ }^{26}$, sino que también a tener mecanismos para poder disminuir el poder o las capacidades relativas de poder de otros países, estableciendo con ello y de manera clara, la posición de líder regional en Sudamérica ${ }^{27}$. Una situación similar ocurría con el pensamiento geopolítico de Argentina de la época, en la cual se percibía a Brasil como el país rival en términos de poder e influencia en la región ${ }^{28}$. Sin embargo, Argentina comenzó a establecer la proyección de sus intereses especialmente en las zonas ubicadas cerca del Estrecho de Magallanes, las islas adyacentes y también en la Antártica ${ }^{29}$. Mientras que para el caso de Chile, el contexto originó en su momento dos aspectos claves dentro de su concepción geopolítica: la proyección de sus intereses en el Pacífico Sur, como también en establecer equilibrios en términos de poder militar con los Estados vecinos de su frontera norte (Bolivia y Perú), debido a las reclamaciones territoriales que efectuaban estos, tomando como punto de partida las pérdidas que sufrieron en la Guerra del Pacífico (1879-1883) ${ }^{30}$.

Pero sin perjuicio de lo mencionado, lo cierto es que al establecer como título una "escuela de pensamiento" en el ámbito de la geopolítica, aquello no solamente resulta ajeno a la realidad teórica, sino que además es una muestra clara de que si

26. Un ejemplo de lo anterior ha sido la constante declaración de Brasil, en el ámbito de su política exterior, de no intromisión de parte de otros actores internacionales, ya sea Estados u organismos internacionales, en la zona de la Amazonia. Asimismo, otro ejemplo fue la planificación y posterior construcción de la actual capital federal del país, Brasilia, siguiendo una lógica geopolítica en su ubicación geográfica, al centro del país.

27. Child (1979), p. 90; y Barton (1997), p. 51.

28. CHILD (1979), p. 91.

29. Dodds y AtKinson (2000), p. 16.

30. Arriaga (2013) p. 17-18; y Child (1979), p. 102-103. 
bien se desarrolló una forma de pensar, no hubo un debate sistemático en los parámetros del pensamiento geopolítico. Es decir, se aprecia ajeno a la realidad teórica, debido a que se construía sobre los mismos preceptos y conceptos derivados de la geopolítica propia de los últimos años del siglo XIX y primera mitad del siglo XX. Por ende, la catalogación de "escuela de pensamiento", no es un fiel reflejo de la constitución o construcción de nuevos preceptos geopolíticos, especialmente cuando aquel "pensamiento" no tuvo un impacto dentro de los centros de pensamiento por antonomasia, como lo son las universidades. Pero aquello, pese a constituirse en una abierta crítica, también tiene que ser evaluada en su contexto. Es por ello que se hace necesario tomar en cuenta el papel que jugaron las Fuerzas Armadas de la región, para la consolidación y discusión de la geopolítica.

El desarrollo del pensamiento geopolítico en la región ya mencionado, se produjo como consecuencia del intercambio de conocimientos entre Oficiales de las Fuerzas Armadas de la región con sus pares alemanes, especialmente en las tres primeras décadas del siglo XX. Y les correspondió a ellos debatir sobre este nuevo concepto que, por aquellos años, podría establecer ciertas bases explicativas sobre el desarrollo de los países, así como también en lo relativo a la protección y obtención de sus intereses nacionales. Es así que la concepción de la disciplina en la región, como consecuencia tanto de la influencia del pensamiento geopolítico alemán, como por la recepción de las instituciones armadas, tuvo como consecuencia que la geopolítica se asociara muy fuertemente a una manera de, o bien lograr bases de desarrollo, o en su defecto contener a las potenciales amenazas que pudiesen significar un peligro a la seguridad. Y en este último aspecto es que se evidencia la concepción estado-céntrica del pensamiento geopolítico militar, debido a que en la mayoría de los casos, la aceptación de una amenaza se derivaba de un actor estatal.

Pero el hecho de que la geopolítica se vinculara directamente con las Fuerzas Armadas en la región, no solamente tuvo una consecuencia en la manera de racionalizar y lograr una eventual expansión del conocimiento, sino que también se observa en la forma en que este conocimiento no fue debidamente expandido, tanto en términos cuantitativos como cualitativos a otros estamentos y niveles de la sociedad. Pero además es necesario destacar que, y siguiendo la línea argumentativa de Phil Kelly, los militares latinoamericanos lograron desarrollar formas para evaluar, desde la visión clásica de la geopolítica, diferentes eventos, tanto nacionales como internacionales, que merecen un reconocimiento y valoración ${ }^{31}$.

31. Kelly (2016), p. 14-16. 
Al revisar lo planteado sobre las escuelas de pensamiento geopolítico en América del Sur, se constata que cerca de la totalidad de los autores sobre temas geopolíticos son militares, y además que estos establecieron sus líneas de desarrollo tomando tanto las perspectivas clásicas de la geopolítica, vinculando sus propias vivencias como también las cualidades, especialmente geográficas, de los países que eran parte. Y es en ese plano en que los militares de Sudamérica pudieron aumentar sus perspectivas geopolíticas. Por ejemplo, se destaca en la mayoría de los escritos geopolíticos esgrimidos por el segmento militar, la necesidad de ocupar aquellos "espacios vacíos" que tenían los países de la región, especialmente aquellos con un gran territorio despoblado. Lo anterior se complementa con la preocupación por establecer la presencia del Estado en determinadas localidades que, por consecuencia de los análisis geopolíticos, deberían tener un trato de carácter estratégico, como por ejemplo los pasos bioceánicos, ciertos archipiélagos o islas. Otro de los puntos donde el pensamiento geopolítico militar en la región tuvo un énfasis, fue en aprovechar las condiciones geográficas para que, de aquella forma, se pudiese lograr un desarrollo en el mediano a largo plazo. Y finalmente, la visión de la geopolítica clásica llevó a que se establecieran nociones sobre las cuales los países debían tener espacios de expansión y/o proyección, en los cuales su dominio sobre determinadas áreas geográficas debía ser superior, con el objetivo de resguardar los intereses de un país por sobre otro ${ }^{32}$.

Uno de los elementos relacionados a la eventual "proyección” de los países de América del Sur, siempre en un ámbito geopolítico, es la generación y construcción de imaginarios geopolíticos, teniendo como base una eventual merma de los intereses nacionales de un país en favor de otro ${ }^{33}$. Al respecto, y siguiendo el planteamiento de Klaus Dodds, la geopolítica en América del Sur en particular, y en América Latina en general, tuvo una connotación altamente expansionista e imperialista, pese a que se advierten diferencias en la forma en que los conceptos geopolíticos clásicos fueron adaptados ${ }^{34}$. Uno de los ejemplos más reiterados dentro de este plano, es el análisis que se la hace a la obra de Augusto Pinochet, Geopolítica, tomando en cuenta que si bien el autor no toma una posición sobre una escuela de pensamiento

32. Al respecto, es posible observar lo anterior tanto en las obras de militares brasileños, argentinos y chilenos. Y en el caso de estos dos últimos, las concepciones geopolíticas cruzadas llevaron a una serie de incidentes, especialmente en la zona del canal Beagle, cerca del Estrecho de Magallanes, en la década del 50.

33. CHILd (1979), p. 109.

34. Dodds (1993), p. 363-364. 
clásica determinada, lo cierto es que su concepción sobre la geopolítica, lleva a que la disciplina se comprenda desde una base orgánica y expansionista, lo que hace entender al Estado como un verdadero ser vivo, más que como una institución ${ }^{35}$. Pero aquello también podría explicarse como consecuencia de la fuerte influencia del contexto político e ideológico propio del conflicto bipolar, tomando en cuenta los años en que fue escrito el mencionado libro (1968).

Durante los años de la Guerra Fría, la geopolítica fue casi de conocimiento exclusivo de ciertos círculos en la sociedad. El desarrollo de aquellas concepciones geopolíticas, tuvieron su auge en las Escuelas Superiores o Academias de Guerra de las Fuerzas Armadas de la región, y específicamente en los ejércitos y armadas, donde el dominio de la geopolítica era considerado clave dentro de los programas académicos relativos a la formación de los futuros Oficiales de Estado Mayor. Al respecto, si bien hubo escritos que fueron de alcance general para la población de concepciones geopolíticas, aquello se dio con especial fuerza en tiempos en donde el segmento militar tenía un alto nivel de influencia política, debido a los diferentes golpes de Estado perpetrados en la región. Así, pese a que se intentó por aquellos años difundir en algo la visión geopolítica de los militares, los quiebres sociales entre el nivel político y el nivel militar, generó como consecuencia que la geopolítica no fuese bien recibida. Aunque aquella visión estuvo más marcada en los países del cono sur de Sudamérica, en comparación con aquellos de la región andina o Brasili ${ }^{36}$.

Uno de los puntos clave, es comprender que en las Escuelas Superiores o Academias de Guerra, es donde no solamente se conforman los futuros cuadros de comandantes y generales dentro de las diferentes Fuerzas Armadas, sino que también es donde se debaten los principales puntos y aspectos que se relacionan con la toma de decisión, dentro del nivel estratégico. Es decir, aquellos Oficiales que luego estarán en posiciones de decisión en el ámbito estratégico, adquieren su formación doctrinaria y teórica en dichos centros de formación. Por dicha razón, es relevante enfatizar el rol de los centros de formación citados y su vinculación con la geopolítica, ya que es en aquellos centros en donde los Oficiales establecían -y establecen- su matriz de pensamiento geopolítico, la que luego colocaban en la práctica, especialmente en momentos en donde el nivel estratégico se mezclaba con el nivel político. Lo anterior sucedía especialmente en los contextos de golpes de Estado.

35. BARTON (1997), p. 64.

36. No obstante lo mencionado, existieron ciertos espacios en donde la vinculación entre civiles y militares en torno al conocimiento geopolítico, se produjo, aunque el alcance del mismo por aquellos años fue reducido. 
Sobre lo señalado, es posible evidenciar que entre los años 1950 y 1980, el debate geopolítico mayoritariamente ${ }^{37}$ estuvo marcado por autores vinculados a las Fuerzas Armadas, no dando cabida a la intromisión de la universidad en términos generales. Esto se refleja además al considerar que si bien la geopolítica podía ayudar a "explicar" algunos fenómenos de características tanto nacionales como internacionales, lo cierto es que dentro de la perspectiva militar, la geopolítica era considerada como una herramienta que permitía mejorar los niveles de seguridad dentro del Estado. En otras palabras, la geopolítica era tomada en cuenta como un asunto que ayuda a la seguridad nacional, un aspecto en donde los civiles, incluso históricamente, han tenido un papel muy marginal en comparación con las planificaciones militares a nivel estratégico. Es por eso que, dentro de los textos clásicos de geopolítica de autores militares, se observa una fuerte inclinación en unir a la geopolítica con una perspectiva teórico-realista, propia de la teoría política y de las relaciones internacionales, la cual pone énfasis en el self-help que deben considerar los Estados para poder sobrevivir, frente a un sistema internacional anárquico. Lo cierto es que aquella visión no es precisa, debido a que se confunde una base teórica con una concepción epistémica de la geopolítica, con lo que se pierde el mismo objeto de estudio de la disciplina. Es por eso que aquel debate, el cual se encuentra pendiente, ya ha comenzado a esgrimirse, especialmente desde la academia, especialmente a fines del siglo XX.

\section{El necesario debate geopolítico en la región en la actualidad}

El fin de la Guerra Fría no solamente marcó un hito dentro de la composición económica y política de los sistemas internacionales, sino que también estableció un cambio en la manera de comprender los fenómenos, desde el punto de vista de la academia. En este sentido, la realidad de la nueva etapa que se comenzaba a evidenciar no podía ser explicada ni entendida desde los mismos parámetros conceptuales propios de aquel período de la historia. Y si a lo anterior se le agrega la visión clásica de la geopolítica, las consideraciones analíticas y explicativas se complejizan aún más, producto que su derivación epistémica proviene de fines del siglo XIX. Por lo tanto, se hizo necesaria una nueva conceptualización de la disciplina, que ayudara a comprender otros fenómenos que se están replicando en la

37. Una excepción a lo anterior representa el caso de Brasil, en el cual es posible encontrar autores civiles que aportaron al conocimiento geopolítico, aunque también tuvieron su vinculación con el estamento militar. 
región. Al respecto, fenómenos como la globalización, el mismo reordenamiento de fuerzas políticas y militares luego de la caída del Muro de Berlín, como también la aparición de fenómenos provenientes de actores no convencionales, pero que colocaban en riesgo a la seguridad de los países, eran algunos de los aspectos con los cuales las visiones clásicas chocaban en ofrecer una explicación dentro de sus parámetros teóricos, especialmente en el ámbito de la geopolítica.

Es así que, reconociendo el mencionado contexto, Detlef Nolte y Leslie Wehner señalan de forma explícita algunos de los principales factores que, desde el fin del conflicto bipolar, influyen en la generación del pensamiento geopolítico en la región. Entre ellos es posible mencionar un cambio en la forma en que la región se ha proyectado hacia el resto del sistema internacional, dejando de lado una concepción de "aislamiento" de los acontecimientos de relevancia mundial. También consideran los diferentes proyectos de integración regional dentro de Sudamérica, que aportan tanto a la concepción de región que se desea, como también a los elementos sobre los cuales se construye la integración. Y finalmente, cabe destacar la importancia que para Sudamérica poseen los recursos naturales, tanto como base para mantener modelos de desarrollo, como también causantes de problemas entre los países de la región ${ }^{38}$.

A comienzos del siglo XXI, la región sudamericana sigue siendo considerada marginal a la hora de establecer aspectos de relevancia o de prioridades, en la agenda de seguridad internacional a nivel mundial. Pero dicha visión no significa que la región se haya marginado de las prioridades de dicha agenda. Es así como la concepción de "nuevas amenazas", entre ellas el terrorismo, el narcotráfico, las consecuencias sociales negativas que generan diferentes fenómenos de la naturaleza, han aportado a la redefinición de los parámetros de seguridad, especialmente cuando no es un Estado el actor que se convierte en un factor de amenaza, e incluso más, cuando muchas amenazas para la seguridad de las sociedades tienen su origen al interior de las mismas ${ }^{39}$. Por lo tanto la geopolítica, desde la concepción Estadocéntrica, no permite dar explicaciones palpables sobre dichos fenómenos.

Algo similar ocurre con los diferentes proyectos de integración que se han establecido a nivel regional. La cercanía de posiciones, tanto políticas como económicas entre países que, para la visión de la geopolítica derivada de la Guerra Fría, eran considerados rivales, ha dado como resultado que la cooperación sea uno de

38. Nolte y Wehner (2016), p. 35.

39. GRIFFITHS (2007), pp. 92-95. 
los elementos que determine la integración entre los mismos, dejando de lado las eventuales diferencias que se puedan suscitar. Y pese a que aún existen focos en donde el territorio sigue siendo un elemento de división entre Estados, como en los casos de Chile con el Perú, los diferentes niveles de cooperación e integración ha llevado que la probabilidad de un conflicto de carácter convencional, sea baja. Esta visión, de acuerdo a Rivarola, es propia de un entendimiento que se da a través de la explicación de una geopolítica basada en las nuevas estructuras de regionalismo e integración en Sudamérica, algo que no era palpable ni visible durante la época del conflicto bipolar ${ }^{40}$.

Si bien es cierto que los aspectos que señalan tanto Nolte como Wehner, han sido objeto de un planteamiento geopolítico, este se ha quedado enfocado desde la perspectiva clásica de la disciplina, dando muy poca o nula cabida a otro tipo de enfoques que, pese a tener el rótulo de geopolíticos, no son considerados como tales desde la visión más ortodoxa de la disciplina. Un ejemplo de lo señalado es que en la actualidad, existen una serie de fenómenos que si bien pueden ser considerados desde la óptica de la geopolítica, la concepción clásica de la disciplina no permite una adecuada interpretación, explicación o análisis de los mismos. Eso se debe a que las visiones geopolíticas para realizar aquello, tomando el marco establecido durante el conflicto bipolar, se basa en una única raíz epistémica, lo que no permite ir más allá ${ }^{41}$. Es así como conceptos como territorio, identidad e incluso la misma visión de la disciplina han ido evolucionando de la mano con los nuevos debates dentro de las Ciencias Sociales, los cuales merecen un tratamiento especial.

La visión clásica de la geopolítica es eminentemente territorial. Aquello se visualiza no solamente en la forma de establecer sus diferentes perspectivas teóricas con respecto a los Estados, sino que también en la forma en que el territorio, para muchos de los autores clásicos, es una clara representación del poder de los países. Así, la pérdida o ganancia de territorio, es un factor determinante del poder que un Estado posee. Por lo tanto, el territorio se constituye como un absoluto dentro de un juego de suma cero, lo cual no da espacio para una comprensión mucho más amplia de la totalidad de elementos que se involucran

40. Rivarola (2011), pp. 858-860.

41. Al respecto, Detlef Nolte y Leslie Wehner señalan que si bien en América del Sur se ha establecido una tradición y desarrollo del pensamiento geopolítico clásico, sus concepciones más contemporáneas, como la geopolítica crítica, no ha tenido el mismo tratamiento. Véase para mayor información Nolte y WeHner (2016) p. 36; Kelly (2016), p. 15. 
dentro del territorio, e incluso de los aspectos que explicarían una modificación de la percepción del territorio en $\mathrm{si}^{42}$.

Otro de los aspectos absolutos dentro de la concepción del territorio es la vinculación directa que se tiene del mismo con la noción de Estado-Nación. Como ya se señaló, la geopolítica clásica se encuentra asociada, de manera muy general, a la visión teórica del realismo, especialmente desde la perspectiva de la disciplina de las relaciones internacionales. Lo cierto es que dentro de los preceptos básicos del realismo, se encuentra la interpretación del Estado-Nación como un ente unitario, en el sentido de que no existe otro poder o unidad dentro del Estado que pueda tener un nivel decisional similar a las autoridades centrales al mismo. Desde aquella perspectiva, cualquier intento de secesión dentro del Estado, o bien una pérdida territorial a favor de otro actor estatal, es tomado como una disminución de las capacidades materiales y relativas del Estado. Pero a lo anterior, es necesario añadir que el territorio para dicha visión teórica, se aprecia como algo homogéneo, con la excepción de aquellos sectores que pudiesen revestir un interés de características estratégicas, siempre desde el punto de vista del poder central de la unidad estatal.

La actualidad no se condice con el contexto mencionado en el párrafo anterior. Los medios masivos de comunicación, así como también la posibilidad de conocer y comprender fenómenos que se encuentran, desde un punto de vista geográfico, a miles de kilómetros de distancia, en tiempo real, no solamente han modificado la realidad y comprensión del territorio, sino que además lo han moldeado para poner en entredicho, conceptos tales como el de soberanía al interior del Estado. Pero incluso se va más allá. El territorio como un absoluto, como un aspecto que no tiene capacidad para moldearse a las realidades sociales cambiantes, no es tal. Es por eso que resulta, desde una perspectiva más amplia, considerar conceptos como el de "espacio" por sobre el de territorio, ya que se toman en cuenta tanto las diferencias que hay entre territorios, como también los elementos que moldean espacios e identidades dentro de los mismos, lo que genera como consecuencia la necesidad de considerar el rol de las localidades y los discursos, para comprender un fenómeno de características geopolíticas en la actualidad ${ }^{43}$.

42. Una visión del territorio como símbolo de poder, se visualiza desde la perspectiva realista, donde se considera un elemento dentro de un juego de suma cero, donde el país que adquiere un territorio, perjudica a otro, no importando si dicho territorio pudiese considerarse como un área de cualidades estratégicas o no.

43. Agnew (1994), p. 56-57. 
Relacionado con la concepción anterior, las identidades dentro de la conceptualización clásica de la geopolítica, no poseen un peso mayor que determine un cambio o un mejor entendimiento de una realidad o proceso geopolítico. Es así que para la concepción clásica, las realidades identitarias vienen dadas directamente por las cualidades geográficas que pueda tener un país, siempre en directa relación con el contexto en el cual se desenvuelve, la extensión de su territorio y la historia que pueda compartir con los Estados colindantes. Por lo tanto, un país establece una identidad no tomando en cuenta los pequeños relatos o discursos que son, de manera efectiva, generadores de identidades tanto nacionales como regionales o locales, pero la visión clásica de la geopolítica no coloca énfasis en aquello, lo que va en contra de los procesos geopolíticos de la actualidad. Es así como la gran mayoría de los procesos de autodeterminación en el presente siglo, se han levantado sobre la base de una identificación de pequeños relatos que establecen diferencias con respecto a otras identidades, tanto a nivel nacional como local ${ }^{44}$. Es por eso que autores que pueden ser identificados como seguidores de una concepción clásica de la geopolítica, han tomado conciencia de que dichos elementos no pueden ser dejados de lado, e incluso deben ser priorizados, al momento de comprender y explicar un proceso geopolítico ${ }^{45}$.

Lo anterior además se complementa con el hecho de que el territorio no puede considerarse como un aspecto homogéneo, especialmente cuando se abordan aspectos identitarios. Las localidades, especialmente aquellas que tienen un grado de vinculación fronteriza, poseen realidades muy diferentes a aquellas zonas que se encuentran lejanas a la interacción en términos de fronteras. La visión de la geopolítica clásica, al establecer en un sentido general al territorio como un absoluto, no solamente deja de lado aquellas perspectivas relacionadas con las localidades, y sus respectivas diferencias entre las mismas, sino que dentro de su relato, la identidad local presenta un desafío para la mantención de los intereses del Estado como un todo, especialmente cuando el "territorio" se visualiza como un concepto aglutinador.

44. FonT y Rufí (2001), p. 19-20.

45. Al respecto, autores como Robert Kaplan y Zbigniew Brzezinski, consideran que la cultura y las identidades locales, que en múltiples ocasiones van más allá de la identidad nacional de carácter estatal, son más relevantes para comprender tanto el origen de los conflictos que en la actualidad afectan a la seguridad internacional, como también a su eventual solución. Aunque lo anterior no hace que se aparten de su visión realista en su mayoría. Véase KapLan (2012) y BRZEZINSKI (1998). 
Es por eso que, en un sentido amplio, la geopolítica no puede tomarse como una disciplina única y sin variaciones. Una concepción amplia de lo que es el territorio, derivando en la importancia del espacio, en conjunto con un punto de vista donde las identidades se colocan al frente de una noción Estado-céntrica absoluta, da como resultado nuevas nociones sobre la identificación de los procesos geopolíticos en el mundo, pero con una conceptualización mucho más cercana a una realidad social. Así, la concepción crítica de la geopolítica no puede ser comprendida, sin tomar en cuenta los parámetros clásicos de la disciplina y viceversa, si se desea otorgar un valor y un desarrollo a los preceptos geopolíticos ${ }^{46}$. Sin embargo, aquello solamente se visualiza teniendo una base que va más allá de la concepción epistemológica positivista de la disciplina; por lo tanto, lo anterior se lograría solamente debatiendo los lineamientos que se han producido dentro de las Escuelas Superiores o Academias de Guerra de las Fuerzas Armadas, incorporando con ello visiones desde diferentes puntos de vista. Siendo así, lo anterior lleva a una necesaria discusión con otras disciplinas, las cuales poseen su fundamento en la academia, en la universidad.

Al respecto, cabe mencionar que los preceptos más contemporáneos de la geopolítica, como es el caso de la geopolítica crítica, han sido considerados dentro de la disciplina, especialmente en términos comparativos con la perspectiva clásica. Sin embargo, en algunas ocasiones, la interpretación que se le otorga a dicha rama de la geopolítica no es la adecuada, valiéndose de un desconocimiento como también de un sesgo sobre los alcances y concepciones del mundo (y sus procesos) ${ }^{47}$. Aquello no es más que un reflejo de la necesidad no solamente de tener una concepción mucho más amplia de lo que representa, hoy por hoy, la geopolítica como disciplina, sino que también en lo relativo a la conformación de una verdadera comunidad epistémica sobre aquella forma de evidenciar y explicar diversos fenómenos mundiales.

\section{Conclusiones}

Pese a que Sudamérica ha sido laboratorio para la experimentación de las visiones clásicas de la geopolítica, aquello posee una relación directamente inversa en términos de proporciones con respecto a los nuevos parámetros de la disciplina. ¿Por qué se da este fenómeno? De acuerdo a lo mencionado aquí, aquello puede

46. Dodds (2000), p. 31 y Flint (2006), p. 16.

47. Contreras (2007), p. 42-43; y Le Dantec (2007), p. 72-73. 
ser entendido como la carencia de comunicación y generación de conocimiento mutuo entre universidades y aquellas instituciones que han desarrollado la visión geopolítica, de manera histórica, como lo son las Fuerzas Armadas. Pero además, eso puede tener una fuerte relación con el desconocimiento real que se posee de los nuevos lineamientos que se han dado en el ámbito de la geopolítica, como es el caso de la geopolítica crítica. Pero lo cierto es que más allá de alguna eventual hipótesis, existe un déficit de conocimiento geopolítico como un todo, el cual involucre un desarrollo epistémico, teórico y metodológico de la disciplina desde la región.

Pero aquello que si bien puede ser interpretado como una debilidad dentro del conocimiento geopolítico, también puede ser visualizado como una verdadera oportunidad, en el sentido de que, a través de la geopolítica, es posible la construcción de canales de comunicación entre las Fuerzas Armadas y los centros de educación superior, que permitan la generación de una masa crítica, ayudando con ello al desarrollo de nuevos puntos de vista sobre la geopolítica. Así, la geopolítica puede servir de canal de comunicación y de mejora del entendimiento entre los centros de educación superior y las Fuerzas Armadas, en torno a un objetivo común, el cual se resume en un mejor desarrollo y discusión de los alcances de la geopolítica.

Sin embargo, tampoco se puede desconocer el hecho de que la región, Sudamérica, se encuentra un tanto rezagada en la comprensión de las nuevas perspectivas de la geopolítica, incluso desde el punto de vista académico. Es por eso que resulta una necesidad contar con la debida actualización de los conocimientos geopolíticos, con el fin de poder tener una mejor comprensión y explicación de los diferentes fenómenos en que la región se ve involucrada, como por ejemplo las denominadas "nuevas amenazas", los diversos procesos de integración, así como también hacer visibles las múltiples identidades que se encuentran en el espacio territorial de la región. Así, tanto las oportunidades como amenazas que se encuentran en Sudamérica, podrían ser comprendidas de una forma más holística y, por ende, se encontrarían más susceptibles a ser aprovechadas o combatidas.

\section{Referencias bibliográficas}

Agnew, John (1994): “The Territorial Trap: The Geographical Assumptions of International Relations Theory". En Review of International Political Economy $\mathrm{N}^{\mathrm{o}}$ 1, Vol. 1, pp. 53-80.

Arriaga, Juan Carlos (2013): El largo proceso histórico de partición territorial. 
Las fronteras en América Latina y el Caribe, siglos XVI al XXI (Ciudad de México, Bonilla Artigas Editores - Universidad de Quintana Roo).

Barton, Jonathan (1997): A Political Geography of Latin America (New York, Routledge).

BRZEZINSKI, Zbigniew (1998): El gran tablero mundial. La supremacía estadounidense y sus imperativos geoestratégicos (Barcelona, Paidós).

CABRERA, Lester (2017): "La vinculación entre geopolítica y seguridad: algunas apreciaciones conceptuales y teóricas". En URVIO, Revista Latinoamericana de Estudios de Seguridad No 20, pp. 111-125.

CHILD, John (1979): "Geopolitical Thinking in Latin America”. En Latin American Research Review No 14, Vol. 2, pp. 89-111.

CoHen, Saul (2015): Geopolitics. The Geography of International Relations (New York, Rownman \& Littlefield).

Contreras, Arturo (2007): “Análisis critico de la geopolítica contemporánea”. En Política y Estrategia $\mathrm{N}^{\circ} 108$, pp. 29-45.

Dodds, Klaus (1993): "Geopolitics, cartography and the state in South America". En Political Geography No 12, Vol. 4, pp. 361-381.

DodDs, Klaus (2000): Geopolitics in a changing world (New York, Prentice Hall).

Doods, Klaus y AtKINSON, David (2000): "Introduction to geopolitical traditions: a century of geopolitical thought". En Doods, Klaus y AtKInson, David. Geopolitical Traditions: A century of Geopolitical Thought (New York, Routledge), pp. 1-24.

FuINT, Colin (2006): Introduction to Geopolitics (New York,Routledge).

Font, Joan y Rufí Joan (2001): Geopolítica, Identidad y Globalización (Barcelona, Ariel).

Griffiths, John (2007): "Seguridad Hemisférica en América Latina. Alcances y Proposiciones". En Revista Globalización, Competitividad y Gobernabilidad $\mathrm{N}^{\mathrm{o}}$ 1, Vol. 1, pp. 88-104.

KaPLAN, Robert (2012): The Revenge of Geography. What the map tells us about coming conflicts and the battle against fate (New York, Random House).

Kelly, Phil (2016): Classical Geopolitics. A New Analytical Model (Stanford, Stanford University Press).

Lacoste, Yves (2011): “Las etapas de la geopolítica”. En GonzÁlez Aguayo, Leopoldo. Cuaderno de Trabajo. Antología. Los principales autores de las escuelas de la geopolítica en el mundo (Ciudad de México, Universidad Nacional Autónoma de México), pp. 11-22, 
Le Dantec, Francisco (2007): "Contribución de la geopolítica crítica a la comprensión de la actual concepción de seguridad”. En Política y Estrategia $\mathrm{N}^{\circ} 108$, pp. 71-82.

MÜLler, Martin (2008): "Reconsidering the concept of discourse for the field of critical geopolitics: Towards discourse as language and practice". En Political Geography N $\mathrm{N}^{\circ} 27$, pp. 322-338.

Nolte, Detlef y Wehner, Leslie(2016): "Geopolitics in Latin America, old and new". En Mares, David y Kacowicz, Arie. Routledge Handbook of Latin American Security (New York, Routledge), pp. 33-43.

O'tuathail, Gearoid (1996): Critical Geopolitics. The Politics of Writing Global Space (London, Routledge).

O'Tuathail, Gearoid y Agnew, John (1992): "Geopolitics and discourse: Practical geopolitical reasoning in American foreign policy”. En Political Geography $\mathrm{N}^{\mathrm{o}}$ 11, pp. 190-204.

Rivarola, Andrés (2011): “Geopolitics of Integration' and the Imagination of South America”. En Geopolitics Vol. 16, No 4, pp. 846-864.

TAYlor, Peter y Flint, Colin (2002): Geografía Política. Economía mundo, EstadoNación y Localidad (Madrid, Trama Editorial). 